\title{
Structures in the dust coma of comet C/1999 T1 (McNaught-Hartley) from Jan. 26 to Feb. 05, 2001 (Research Note)
}

\author{
L. M. Lara ${ }^{1}$, J. Licandro $^{2}$, and G.-P. Tozzi ${ }^{3}$ \\ 1 Instituto de Astrofísica de Andalucía, CSIC, PO Box 3004, 18080 Granada, Spain \\ e-mail: Lara@iaa.es \\ 2 Instituto de Astrofísica de Canarias, PO Box 321, 38700 Santa Cruz de La Palma, Tenerife, Spain \\ e-mail: jlicandr@iac.es \\ 3 INAF - Osservatorio Astrofisico di Arcetri, largo E. Fermi 5, 50125 Firenze, Italy \\ e-mail: tozzi@arcetri.astro.it
}

Received 13 May 2008 / Accepted 22 January 2009

ABSTRACT

\begin{abstract}
Aims. An in depth analysis of the images acquired in broad-band optical and infrared observations in the $R, I$, and $J, H, K$ s bands of comet C/199T1 (McNaught-Hartley) acquired from Jan. 26 to Feb. 05, 2001 has been performed and is presented here.

Methods. Beside the Laplace filtering technique applied to enhance structures in the dust coma of the comet McNaught-Hartley, we have also made use of the radial renormalization method to reveal large scale structures, usually broad jets.

Results. We find that, contrary to other work, the comet did indeed show non-spherical structures besides the dust and ion tail. These structures, while not unambiguously detected when applying a Laplace filtering technique with small box sizes, have become evident by making use of radial renormalization techniques and larger box sizes in the Laplace filtering technique. This research note reports on these features.

Conclusions. Geometrical analysis of the features and its evolution allows us to tentatively conclude that either the synodic spin period is very close to $24 \mathrm{~h}$, or it has a rotation period much longer than 10 days, which could be indicative of the nucleus approaching an excited spin state, or the rotation axis is oriented such that an active area is permanently illuminated by the Sun and the jet is pointing toward or away from the observer.
\end{abstract}

Key words. comets: individual: McNaught-Hartley (C/1999 T1)

\section{Observations and data reduction}

Comet C/1999 T1 (McNaught-Hartley) was observed in the near-infrared and in the optical range. The near-infrared and optical observations were made by using three different telescopes: the $3.56 \mathrm{~m}$ Telescopio Nazionale Galileo (TNG), the Italian telescope at El Roque de los Muchachos Observatory (La Palma, Canary Islands, Spain); the $1.5 \mathrm{~m}$ Telescopio Infrarosso del Gornergrat (TIRGO), the Italian infrared facility located in the Swiss alps at $3200 \mathrm{~m}$ altitude; and the $82 \mathrm{~cm}$ IAC-80 telescope of IAC at Teide Observatory (Tenerife, Canary Islands, Spain).

From Jan. 26 to 31, we carried out near-infrared imaging observations with the TIRGO using the ARNICA (ARcetri Near Infrared CAmera) imaging camera covering the near infrared bands between 1.0 and $2.5 \mu \mathrm{m}$. The scale is $1^{\prime \prime}$ per pixel, with sky coverage of more than $4^{\prime} \times 4^{\prime}$ on the detector array, a NICMOS 3 type $(256 \times 256$ pixels, $40 \mu \mathrm{m}$ side $)$ broad-band $J(1.26 \mu \mathrm{m})$, and $H(1.65 \mu \mathrm{m})$ and $K \mathrm{~s}(2.205 \mu \mathrm{m})$ images were obtained every night from Jan. 26 to 31, 2001, but not on Jan. 29 due to bad weather conditions. Near-infrared images in the same bands were also taken on Feb. 4 and 5 with the TNG using NICS (the Near Infrared Camera Spectrograph). The detector is a $\mathrm{HgCdTe}$ Hawaii $1024 \times 1024$ array that, using the large field camera, has a plate scale of $0.25^{\prime \prime}$ per pixel covering a $4^{\prime} \times 4^{\prime}$ field.
The IR observations were complemented with observations in the optical range (broadband $R$ and $I$ filters) on Jan. 26 and Feb. 5. On Jan. 26, the images were taken with the TNG using the camera-spectrograph DOLORES (Device Optimized for the LOw REsolution) and with the IAC-80 telescope using the Thomson $1 \mathrm{k} \times 1 \mathrm{k}$ direct CCD camera. On Feb. 5 the $R$ and $I$ images were taken with the IAC-80 telescope. In imaging mode, DOLORES provides a $9.4^{\prime} \times 9.4^{\prime}$ field $\left(0.275^{\prime \prime}\right.$ per pixel plate scale), while the Thomson CCD at the IAC- 80 provides a $7.3^{\prime} \times 7.3^{\prime}$ field $\left(0.43^{\prime \prime}\right.$ per pixel plate scale).

The acquired data pertain mostly to the sunlight reflected by the cometary dust as the $R$ and $I$ broadband Johnson filter, and the near-IR $J H K$ filter bandpasses cover little contamination by gaseous emissions. Thus, our analysis concerns the dust coma of C/1999 T1 (McNaught-Hartley).

The near infrared images were obtained in the usual way (i.e. chopping between the comet and the sky). In the case of TIRGO observations, for all of the filters, four consecutive or alternate series of 8 images of 15 or $10 \mathrm{~s}$ exposure time in the $J$ band and $5 \mathrm{~s}$ integration time in the $H$ and $K \mathrm{~s}$ bands were obtained during our run. Sky images were also obtained in the same way in all but the tail directions, at a distance of 8 arcmin from the comet. In the case of TNG observations, one series of 7 images of the comet of $10 \mathrm{~s}$ exposure time in the $J$ and $H$ bands and 
Table 1. Details of the observations.

\begin{tabular}{cccccc}
\hline \hline $\begin{array}{c}\text { Date } \\
(2001)\end{array}$ & $\begin{array}{c}\text { Time } \\
\mathrm{UT}\end{array}$ & $\begin{array}{c}\Delta \\
\mathrm{AU}\end{array}$ & $\begin{array}{c}r_{\mathrm{H}} \\
\mathrm{AU}\end{array}$ & $\begin{array}{c}\text { PA } \\
\mathrm{deg}\end{array}$ & $\begin{array}{c}\text { Phase } \\
\text { deg }\end{array}$ \\
\hline Jan. 26 & $06: 34-07: 17$ & 1.298 & 1.355 & 293.2 & 43.5 \\
Jan. 28 & $03: 32-04: 40$ & 1.293 & 1.370 & 293.1 & 43.3 \\
Jan. 30 & $03: 20-03: 49$ & 1.290 & 1.387 & 292.9 & 43.0 \\
Jan. 31 & $03: 20-04: 19$ & 1.288 & 1.395 & 292.8 & 42.8 \\
Feb. 05 & $05: 24-06: 18$ & 1.288 & 1.438 & 291.9 & 41.9 \\
\hline
\end{tabular}

PA and Phase refer to the position angle of the extended Sun-component radius vector and to the Sun-comet-observer angle, respectively. $r_{\mathrm{H}}$ and $\Delta$ are the heliocentric and geocentric distances of the comet during our observations.

one of $20 \mathrm{~s}$ exposure time in the $H$ band were taken on Feb. 4 and one series of 7 images of $10 \mathrm{~s}$ exposure time in the $J, H$ and $K \mathrm{~s}$ band on Feb. 5. $J, H$ and $K$ s sky images were median averaged to create the sky and flat field images used to correct the corresponding comet images. Details of the observations are presented in Table 1 and in Table 1 of Lara et al. (2003). All of the observations were made while tracking on the comet proper motion. All nights but Jan. 27 and 28 were photometric. Several infrared standard stars (Hunt et al. 1998) were observed each night for calibration purposes and in order to better determine the calibration uncertainties. The calibration error was $\sim 5 \%$.

\section{Analysis and results}

In Lara et al. (2003) we searched for structures (jets, fans, etc.) in the coma by applying a Laplacian filtering. No clear non-sperical structures were found.

Ho et al. (2008, private communication) used a similar dataset and found a large structure in the optical images. If their findings and ours (Lara et al. 2003) were correct, the dust coma structures that were present during Dec. 29, 2000-Jan. 02, 2001, as firstly detected by Ho et al. would have disappeared in a time frame of weeks (Jan. 26 to Feb. 05, 2001) as we did not detect any structures in the dust coma of C/199 T1.

Triggered by these contradictory results, we performed a reanalysis of the optical and near-IR comet images aiming at the detection of the same or similar structures using an alternative structure enhancement technique and refined parameter sets of the Laplace filter method used for the same dataset. To enhance the presence of structures in the coma of the comet, we used a radial renormalization technique (see for instance A'Hearn et al. 1986) and the adaptive Laplace filtering technique revealing two structures in the processed images: (i) a clear curved jet pointing to the Sun and spiraling to the north-west (as projected on the plane of the sky) into the anti-Sun hemisphere (see Fig. 1), and (ii) a faint one pointing to the south-west which is barely visible in Fig. 1 and more evident in the Laplace filtered image (see Fig. 2).

We investigated why in Fig. 1 of Lara et al. (2003) the jet structure did not appear. The reason is that we applied the adaptive Laplace filtering technique only to the near-IR images acquired at TIRGO by using a very narrow width for the filter $(3,5$, $7,11)$. This is more appropriate to enhance very confined structures near the nucleus and these were not detected in the near-IR images of the comet. However, when a broader filter width (63, $95,127)$ is applied to the $R, I, J, H$ and $K_{\mathrm{S}}$ images, the large
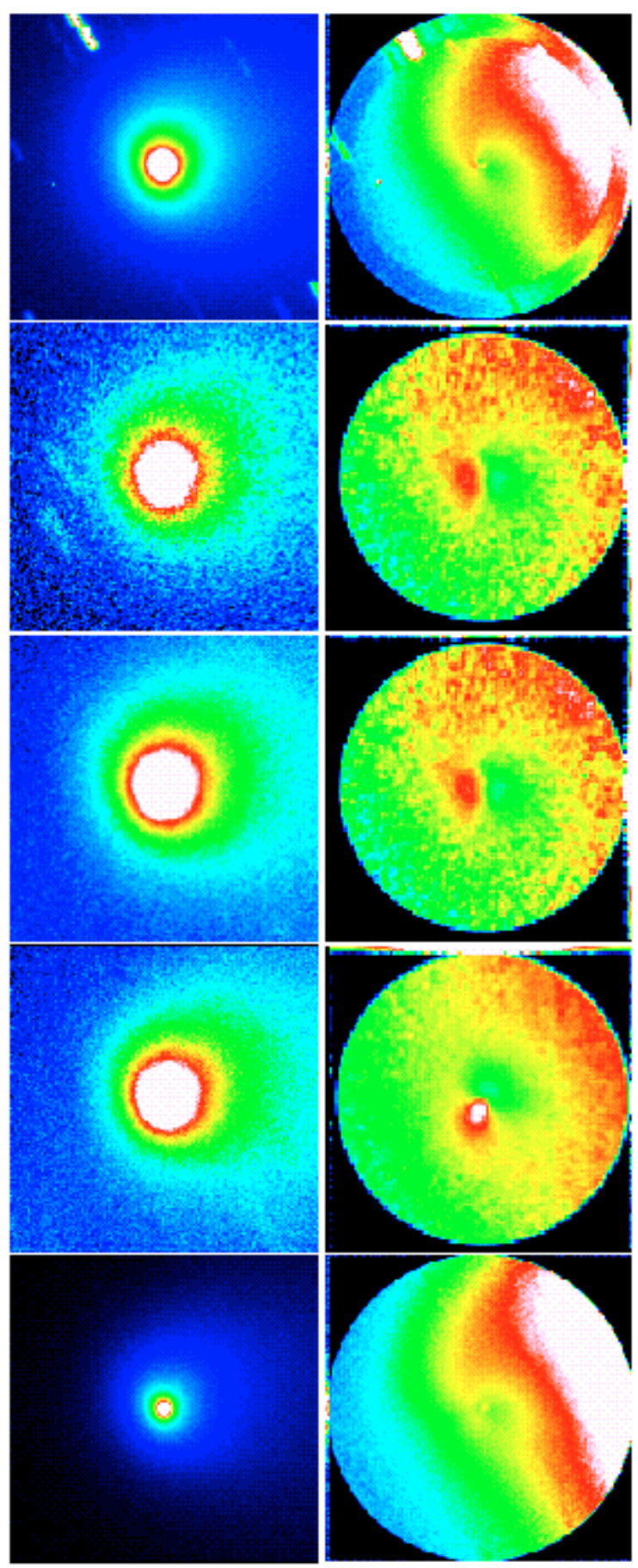

Fig. 1. Reduced and processed images of comet C/1999 T1 from top to bottom, from Jan. 26 ( $R$ Johnson filter), Jan. 28, 30, 31 (in $J$ filter), and Feb. 05, 2001 ( $R$ Johnson filter). The look-up table is linear. North is up and east is to the left. The FOV is $150000 \times 150000 \mathrm{~km}$ for the observed images, whereas for the processed images the FOV is $94000 \times$ $94000 \mathrm{~km}$ in $J$ and $163000 \times 163000 \mathrm{~km}$ in $R$.

spiral jet in the northern hemisphere (see Fig. 1 and A in Fig. 2) plus the narrower and shorter structure pointing in the south-west direction (named B Fig. 2) become clear. The B structure is also enhanced in the radially renormalized $R$ images and we believe it is part of the large spiral jet seen in Fig. 1. This B structure also appears on the $R$ and $I$ comet images when the Laplace filtering technique makes use of a small box-size $(\geq 11)$, whereas it cannot be unambiguously detected in the near-IR images for this small box-size. 

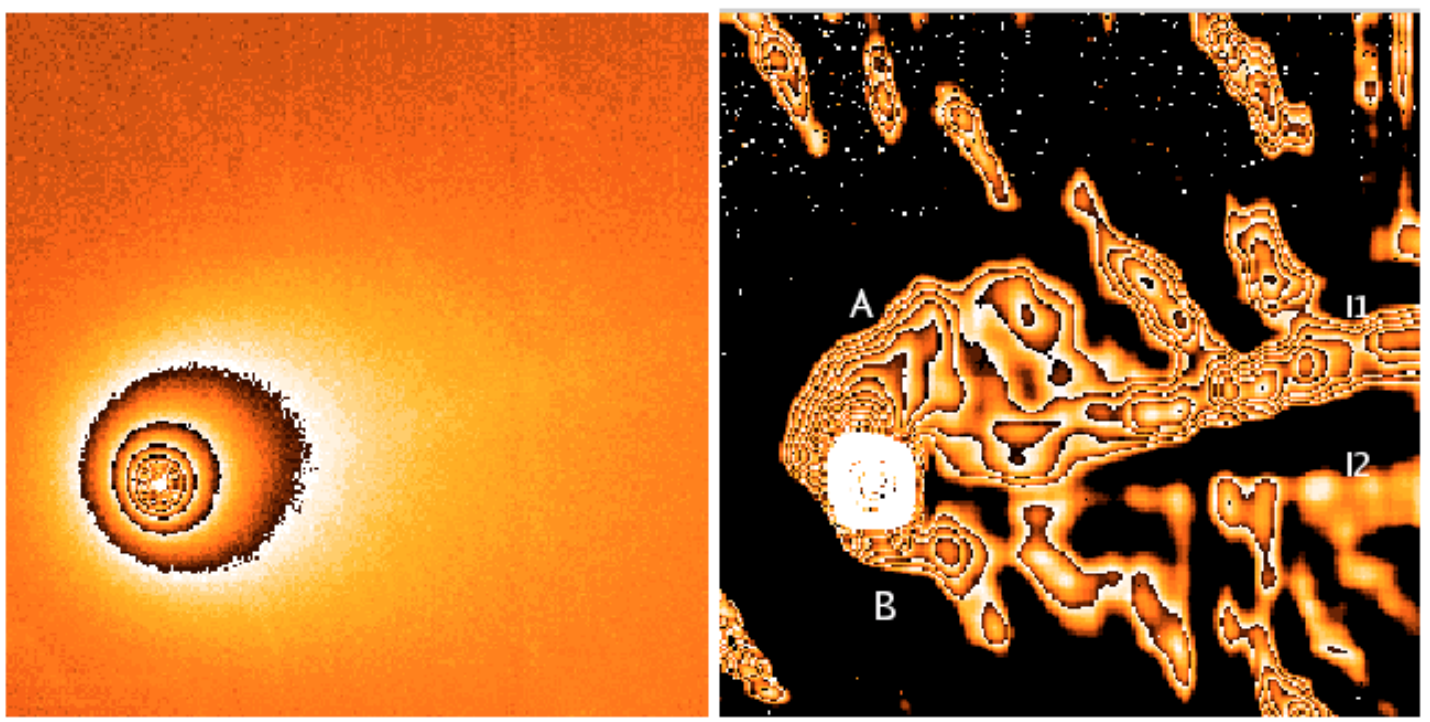

Fig. 2. Flux calibrated image of C/1999 T1 (McNaught-Hartley) on Feb. 05, 2001, with a look-up table that allows us to visualize the isophotes stretching from $1 \times 10^{-12}$ to $3 \times 10^{-8} \mathrm{erg} \mathrm{cm}^{-2} \mathrm{~s}^{-1}$. The processed images are obtained by applying a Laplace filter of 127 pixels width that enhances a broad and large jet in the north hemisphere curved towards the west, another much narrower and shorter in the south-west direction, and two streams of the ion tail in the anti-Sunward direction. Orientation as in Fig. 1. The FOV is $5.76^{\prime} \times 5.76^{\prime}, 320600 \times 320600 \mathrm{~km}$ at the comet distance, for each image.

Furthermore, a very narrow and confined structure I1 in antisunward direction at an angle of $\sim 290^{\circ}$ plus a second fainter streamer $\mathrm{I} 2$ at $\sim 270^{\circ}$ can be seen in Fig. 2. The $R$ filter contains $\mathrm{H}_{2} \mathrm{O}^{+}$emission bands explaining the presence of ion features when processing the images. It similarly happens with the $I$ filter, but to a less extent as its bandpass marginally covers the $\mathrm{H}_{2} \mathrm{O}^{+}$spectroscopic features. The $R$ and $I$ images acquired on Feb. 05 and Jan. 26 display the ion tail being more intense on Jan. 26 than on Feb. 05, 2001.

As our investigation of large and small structures in the dust coma of C/1999 T1 was successful, these structures were compared with those that Ho et al. detected in images of the comet taken 3 weeks before ours. It turns out that structures in Figs. 1 and 2 did also exist during Dec. 29, 2000-Jan. 02, 2001. Furthermore, Ho et al. (private communication) has compared our image on Jan. 26, 2001 and theirs on Dec. 29, 2000, finding that the large structure in the dust coma had not changed within $\sim 1$ month. It has comparable width and direction on both dates. The enhancement of the structure named B in Fig. 2 shows a close dependence on the determination of the optocenter before applying the radial renormalization technique: by artificially shifting the optocenter by \pm 1 pixel, it remains on the enhanced comet image of Jan. 26, 2001, whereas it disappears on the enhanced comet image of Dec. 29, 2000. However, we applied the adaptive Laplace filtering technique to the comet image of Dec. 29 (kindly provided by Ho et al.) and both A and B structures are enhanced. Hence, any interpretation of this feature must be done cautiously.

We have also tried to search for any variation of the jet intensity as well as of the azimuthal position as indicative of comet activity variations and/or of comet nucleus spin period. The images acquired in the near-IR clearly show a lower $\mathrm{S} / \mathrm{N}$ than the optical ones. Thus, our investigation has focused only on the $R$ comet images of Jan. 26 and Feb. 05. Unfortunately, this study has not led to any conclusions as the variation of the jet intensity in the inner coma $(\rho \leq 10000 \mathrm{~km})$, which points to a more intense jet on Jan. 26, disappears when we introduce an error of one pixel in the determination of the optocenter (i.e. $0.275^{\prime \prime}$ ).

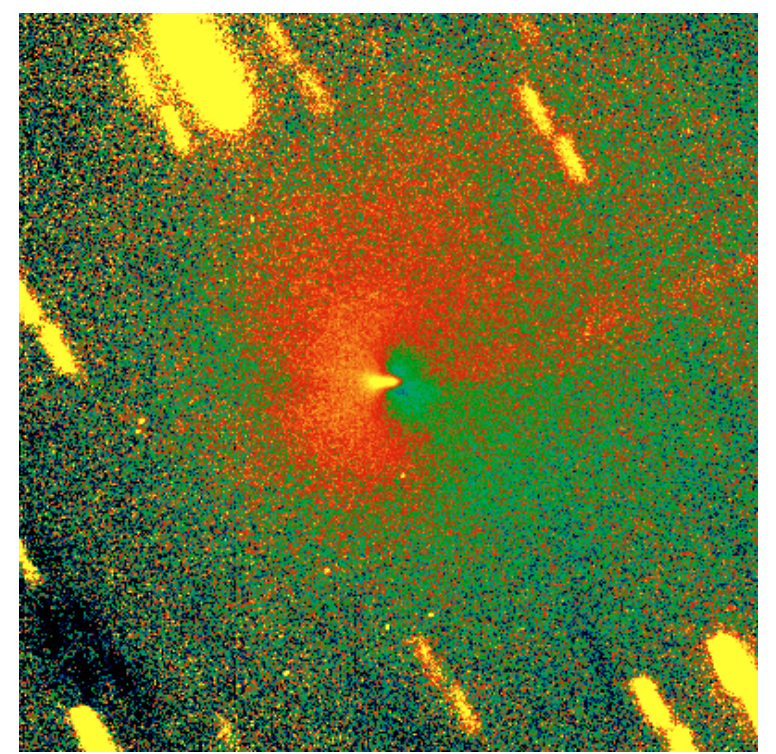

Fig. 3. Dust coma intensity on Jan. 26 with respect to Feb. 05. North is up and East is to the left. FOV is $163000 \times 163000$. Look-up table is linear and it stretches from 0.9 to 1.3 . Note the higher comet activity in the sunward hemisphere and a more prominent ion tail on Jan. 26 versus Feb. 05 .

Furthermore, we studied if the dust comet activity showed any differences between Jan. 26 and Feb. 05, 2001. Beside an overall higher dust comet activity on Jan. 26 (see Table 2 in Lara 2003) the quotient of the flux calibrated comet image on Jan. 26 divided by the one on Feb. 05 , both reduced to $\Delta=r_{\mathrm{h}}=1 \mathrm{AU}$, clearly shows a higher intensity (i.e. dust content) in approximately the sunward hemisphere (see Fig. 3) when the comet was closer to the Sun (that is, on Jan. 26, 2001). This quotient also revealed a more prominent ion tail on Jan. 26 than on Feb. 05. The shape and intensity of this enhanced dust comet activity on Jan. 26 is not sensitive to the optocenter determination within error bars of $\leq 1^{\prime \prime}$, contrary to the case of the dust jet where 
no clear variation of its intensity as a function of time could be determined.

\section{Discussion}

A more thorough analysis of the CCD images in two different spectral ranges (optical and near-IR) of comet C/1999 T1 (McNaught-Hartley) revealed the existence of a dust spiral jet, A, pointing to the north-east and curved down to the west. Beside this large structure, a smaller one, B, to the south-west is enhanced by the adaptive Laplace filter, and is also tentatively seen in the radially renormalized images.

The comet images from Jan. 26 to Feb. 05 were acquired over about $24 \mathrm{~h}$ interval (see Table 1). During those 10 days, the viewing geometry (phase angle) and the position angle of the Sun only varied by two degrees. It is highly regrettable that a longer monitoring was not possible as it could have enabled us to retrieve the rotational parameters of the nucleus by following how this structure changed with time, as has been done for 9P/Tempel 1 by Lara et al. (2006). Nevertheless, the dust coma structures can be tentatively interpreted in terms of the rotational parameters of the nucleus. Bearing in mind that the appearance of the structures does not change over about 10 days, the following conclusions can be drawn:

(i) Inspection of the feature on Jan. 28, 30 and 31 could imply that the rotation period could be an integer divisor of $\sim 24 \mathrm{~h}$ (a similar approach was used by Sekanina 2004, to model the nucleus and jets of comet $81 \mathrm{P} /$ Wild 2). Note that the southern orientation of the structure does not change and the images were taken at time intervals of exactly $24 \mathrm{~h}$. However, when studying the whole data set, this option (integer divisors of 24) has to be excluded as optical images on Jan. 26 and Feb. 05 were acquired $2 \mathrm{~h}$ later than the near-IR: for short spin periods (below $24 \mathrm{~h}$ ), it should be possible to detect changes in the structure geometry, as a significant rotation phase change is involved.

(ii) The spiral jet shows the same geometry on Jan. 26 and Feb. 05. This seems to point to the fact that the rotation period is either 10 days, or much longer than 10 days. If it were 10 days, on day +5 after Jan. 26 (i.e. Jan. 31 ), the rotation phase should be such that the spiral jet should look quite different from the one depicted in Fig. 1. If it were longer than 10 days, it should be much longer than 10 days as the appearance of the structures is very similar on Jan. 26 and Feb. 05, not indicating any change in the rotation phase. Cases of rotation periods much longer than 10 days do occur when the comet nucleus gets to excited spin states. In this situation, the nucleus is able to reach spin periods as long as 200 days (see Gutiérrez et al. 2003). However, if the nucleus spin state were approaching excitation, the rotation period would evolve very quickly. This evolution is inversely proportional to the rotation period meaning that a change of rotational state from a period much longer than 10 days (in pure spin state) to an excited spin state could be verified with images acquired later than Feb. 06, 2001. Unfortunately, we do no have those images to further check this hypothesis.

(iii) Structures in the dust coma of other comets which remain stable (i.e. appearance not altered by the rotation of the nucleus) for days (as was the case for comet C/1996 Q1 Tabur, Lara et al. 2000) have been explained as being produced by an axis rotation position such that there is an active area permanently illuminated by the Sun, regardless the spin period of the comet nucleus, and the jet is pointing toward or away from the observer.

We have also studied variation of the jet intensity and azimuthal position as a function of time. However, no conclusive result has been obtained as the detected variations are highly dependent on the accuracy of the optocenter position. While we are rather confident that the optocenter position can be well determined for images on Jan. 26 and Feb. 05 (errors in the determination of the optocenter are of the order of a pixel fraction by using a 2D Gaussian fit), it is not the case for Jan. 31 . Hence, we can only conclude that the comet showed a higher dust activity, as seen in the optical range, on Jan. 26 than on Feb. 05, 2001. This higher dust activity could also be reflected in the dust jet intensity, however we cannot firmly conclude this.

\section{Conclusions}

The conclusions in Lara (2003) remains valid, excluding the fact that the dust coma of C/1999 T1 does show a curved jet pointing to the north-east and curved to the south-west, another shorter and less intense one pointing in the south-west direction, and another one very faint (but still detectable) to the west. These structures remained stable for the 10 days the comet was monitored, not being possible to detect any obvious variation in the morphology and angular position of the jet from Jan. 26 to Feb. 05, 2001. From this fact, we can draw several conclusions about the rotational state (rotation axis position and spin period) of the comet nucleus, although we cannot confirm any of them as a longer monitoring is needed together with a detailed dust dynamics modeling (as done in Lara et al. 2006; Vincent et al. 2008). These conclusions are:

- Spin period of $\approx 24 \mathrm{~h}$.

- Rotation period much longer than 10 days, which could be indicative of the nucleus getting to an excited spin state.

- Rotation axis such that an area is permanently illuminated by the Sun and spin axis orientation such that the jet is pointing toward or away from the observer.

Acknowledgements. The research carried out has been partially supported by the Spanish Ministerio de Educación y Ciencia under contract ESP2006-02934. Luisa M. Lara acknowledges fruitful discussions with Pedro J. Gutiérrez.

\section{References}

A'Hearn M. F., Hoban S., Birch P.V., et al. 1986, Nature, 324, 649 Boehnhardt H., \& Birkle K. 1994, A\&AS, 107, 101

Gutiérrez, P. J., Jorda, L., Ortiz, J. L., \& Rodrigo, R. 2003, A\&A, 406, 1123

Hunt, L. K., Mannucci, F., Testi, L., et al. 1998, AJ, 115, 2594

Lara, L. M., Schulz, R., Stuewe, J., \& Tozzi, G. P., 2000, Icarus, 150, 124

Lara, L. M., Licandro, J., \& Tozzi, G. -P. 2003, A\&A, 404, 373

Lara, L. M., Boehnhardt H., Gredel, R., et al. 2006, A\&A, 445, 1151

Sekanika, Z., Brownlee, D. E., Economou, T. E., Tuzzolino, A. J., \& Green, S. F. 2004, Science, 304, 1796

Vincent, J. -B., Boehnhardt, H., Bertini, I., et al. 2008, EM\&P, submitted 